\title{
Identifying the Patterns of the Near-synonymous Attributive Verb Pairs in the Academic Written Text
}

\author{
Hu, Hsueh-chao
}

Taiwan

\begin{abstract}
In a piece of an academic written discourse, it is important to present an argument logically and cohesively. The researcher has often to report, evaluate and discuss previous research findings with attributive verbs. It is a great misinterpretation to say that an author 'argues' when what s/he is actually doing is 'report'. The difficulty with using these verbs is that there are many of them, and each of them has a slightly different, and often subtle shade of meaning. For example, the word 'notes' implies a fact but is weaker than 'points out', but they share similar denotative meanings. The present corpus-based study aims to examine the function and usage of the synonymous attributive verbs, hoping to offer insights into expert performance and contribute to classroom practices.
\end{abstract}

Keywords: attributive verbs, academic written discourse, near- synonyms, corpus-based study

\section{INTRODUCTION}

The importance of near-synonyms has received increasing attention in collocational classroom-based research during the past decade or so (Lewis, 2001; Woolard, 2001, Webb \& Kagimoto, 2012). However, it is practically impossible to teach the large number of synonyms due to the limited teaching hours in the classrooms. As a result, the use of corpora has become such a popular tool that the concordance lines can serve as the evidence for learners to observe the different patterns between the synonyms. According to Xiao and McEnery (2006), synonyms or near synonyms are lexical pairs "that have very similar cognitive or denotational meanings, but which may differ in collocational or prosodic behavior. As such, synonymous words are not collocationally interchangeable" (p. 108). In particular, even though two words may share similar cognitive or denotational meanings, they may demonstrate different collocational behaviour. Xiao and McEnery (2006) supported their claims with examples such as strong (tea) vs. powerful (car) and fickle for a negative meaning but flexible with a positive one. Boers and Lindstromberg (2009) offered another example of near-synonyms between rapid and fast. They indicated that the Collins CoBuild online collocation sampler generates no fewer than 14 noun collocates ending in -ion (e.g., succession, deterioration, expansion) with the word rapid but not any of the -ion noun for fast. In the context of collocation learning; however, near-synonyms usually cause L2 learners great difficulty in identifying the pattern differences between them.

In the context of an academic written discourse, it is important to present an argument logically and cohesively. The researcher has often to report, evaluate and discuss previous research findings with attributive verbs. The language of attribution, according to Hunston (1995), can be used to "hedge a statement, to introduce information which corroborates the writer's own view, to indicate gap in research, or to set up a point of view against which the writer wishes to argue" (p. 133-134). It is a great misinterpretation to say that an author 'argues' when what $\mathrm{s}$ /he is actually doing is 'report'. Such disconnection confuses the reader and further compromises the quality of the writing. Moreover, some attributive verbs are neutral whereas others are strong or weak. Introducing a strong argument with a weak or neutral attributive verb leads to contradiction of the piece of writing. Thus, mastering the types of attributive verbs "enhances the coherent flow of the presentation and avoids the monotony from overreliance on repetitive use of verbs like says" (Jabulani, 2014, p. 4). The difficulty with using these verbs, in particular for non-native writers, is that there are many of them, and each of them has a slightly different, and often subtle shade of meaning. Two attributive verbs may have the same denotative meanings but differ in the degree or density of that meaning. For example, the word 'notes' 
implies a fact but is weaker than 'points out', but they share similar denotative meanings (Jabulani, 2014). The present corpus-based study aims to examine the function and usage, including their syntactical patterns and collocations of three pairs of synonymous attributive verbs, hoping to offer insights into expert performance and contribute to classroom practices.

\section{METHODS}

This study aims to compare and contrast the function and usage of some frequently used attributive verbs with similar meanings (e.g., claim vs. maintain, account for vs. explain, answer vs. respond) by examining the academic texts in the field of applied linguistics in a self-created corpus with 507,515 words. The data is the collections of 86 pieces of research papers from two peer-reviewed academic journals (i.e., Language Teaching Research and Language Learning). The journal articles were chosen on the basis of research with original data. The papers are the most recent ones within the last seven years (2009-2015). Derived from a list at the writing center of the University of Texas, the attributive verb pairs chosen for comparison occurred at least 10 times out of the corpus and appeared in no less than 5 or more texts. A qualitative analysis was conducted using the concordance lines to study the textual examples. In other words, the data were manually examined to identify the patterns of functions and use of semantic prosody between the synonymous attributive verbs.

\section{RESULTS}

The results analyze three pairs of synonymous verb pairs: CLAIM vs. MAINTAIN, ACCOUNT FOR vs. EXPLAIN, and ANSWER vs. RESPOND. The findings suggest that these synonymous attributive verbs share similar meanings, but their use and function investigated in the preliminary analysis vary from a small to a considerable extent.

\section{CLAIM vS. MAINTAIN}

An examination of the concordance lines reveals the following:

1. The most common patterns are verb + that-clause, followed by the formulaic use as someone claims, followed by verb + to-infinitive.

2. CLAIM is mostly used with an active voice and occasionally with a passive voice.

3. CLAIM takes both human and non-human entities (e.g., theory, work, and hypothesis) as subject.

CLAIM typically construes disagreement between attributor and speaker, as the following examples show:

(1) The results do not support the claim of skill learning theory (Anderson, 1993), which claims that procedualized knowledge involves different sets of skills for comprehension and production.

(2) Qian et al. (2009) reported a gradual reduction in teacher code-switching in primary schools over four grades, and claimed that this progression benefited the students; however, the evidence they provide for acquisition is largely anecdotal.

The first attributed statement was rejected by the attributor's own research findings and the second was negatively evaluated due to its unreliable source of evidence. However, as Hunston (1995) noted when the text only reflects on rather than constitutes the argument, the statement attributed by CLAIM is not always negatively evaluated by the attributor, as the following example shows:

(3) Krashen's input hypothesis $(1982,1985,1998)$ claimed that production serves only for generating comprehensible input from the interlocutor, and that output does not make a real contribution to acquisition.... Swain (1985), and Swain and Lapkin (1995), on the other hand, argued strongly that 'pushed output' leads the learner to notice L2 elements and modify their output, both of which contribute to acquisition.

The example above reveals that though there is a clear disagreement between what Krashen's hypothesis said and what Swain and associates believed, the attributor does not partake in the disagreement. In addition, there are also occasions where the statement attributed by CLAIM is positively evaluated by the attributor, as the following example shows:

(4) With regard to the different factors that have been studied in connection with incidental L2 vocabulary acquisition, Hulstijn (2003) claimed that findings show that "it is elaboration of or 
involvement in the lexical information being processed rather than any of the factors per se that determines retention" (p. 364). In effect, empirical evidence seems to support the proposition that "more meaningful processing is usually associated with higher levels of recollection" (Craik, 2002, p. 316).

The attributor evaluates the attributed statement positively by citing other evidence in support of the claim.

In sum, while CLAIM usually construes disagreement between speaker and attributor, it is not always the case. Sometimes the evaluation of attribution is evident when the co-text and context of node word extend widely enough for making such judgment.

\section{MAINTAIN}

An examination of the concordance lines reveals the following findings:

1. The subject position of MAINTAIN can be either human (e.g., authors, we, and they) or nonhuman entities (e.g., theories of lexical network and the strong interface position). Interestingly, while there is no first person pronoun taking the subject position of CLAIM, there is ' $w e$ ' as the subject of MAINTAIN.

A survey of the concordance lines with their extended co-text and context reveals neither explicitly negative nor positive attributor judgment. That is, most of the statements attributed by MAINTAIN are evaluated as neutral, as the following examples show:

(5) Several second language (L2) acquisition researchers (Long, 1996; Long \& Robinson, 1998; Robinson, 1995, 2001, 2003; Schmidt, 1990, 1995, 2001) maintain that attention to form plays an important role in learning an L2. Schmidt $(1990,1995,2001)$, in particular, has claimed that it is the conscious noticing of the formal aspects of an L2 in the input that allows learners to gain awareness of the target forms, which in turn helps them to monitor the accuracy of their language production.

(6) Studies of encoding variability in human memory research were initially developed in response to the associationist framework of verbal learning which maintained that repeated presentations of items increases recall performance by increasing the strength of association among the items (Martin, 1968; Winograd \& Geis, 1974). In contrast to this perspective, other researchers (Hintzman, 1976) suggested that repeated presentations function to increase the inter-associations among elements or components of the target items.

It can be observed that the use of MAINTAIN in the examples above does not construe any disagreement between speaker and attributor. In the first example, the attributed statement provides the ground for the attributor to develop more detailed description of 'noticing'. In the second example, thought the attributor is presenting a contrasting statement made by other researchers, no explicit attributor judgment can be found in the co-text.

To conclude, CLAIM and MAINTAIN are both attributive verbs that have shared similar denotative meanings. Yet their use and function investigated in the preliminary analysis varies considerably. While CLAIM typically construes conflict between speaker and attributor, the evaluation of statement attributed by MAINTAIN is typically neutral. Also, it is found that MAINTAIN may take first person pronoun as subject, thought there is only one instance.

\section{ACCOUNT FOR vs. EXPLAIN}

The verb phrase ACCOUNT FOR has a normalized frequency of 68 and a range of 34; EXPLAIN, 192 and 69.

Since the raw frequency of ACCOUNT FOR in the corpus was only 69, all of its concordance lines were examined. On the other hand, 100 randomly-selected concordance lines of EXPLAIN was chosen for analysis. The overall results were presented as follows:

1. While EXPLAIN sometimes takes human subjects, ACCOUNT FOR hardly does so.

2. Both verbs were used in active and passive voice as well. 
Judging from the frequency and range statistics alone, it's clear that while the two verbs are synonymous, EXPLAIN is more frequently and widely used than ACCOUNT FOR. A frequentlyrepeated pattern for both verbs is used to report the results of regression analysis, as shown in the examples below. In this case, they seem to be completely interchangeably.

(1) The first residual contrast accounted for $2.2 \%$ of the variance (eigenvalue $=5.4$ ).

(2) In these data, the Rasch model accounted for $38.0 \%$ of the variance (eigenvalue $=92.0$ ), a figure that matched what would be expected if the data fit the Rasch model perfectly.

(3) This variable accounted for $37 \%$ and $24 \%$ of the variation in the gains made in reading and listening, respectively.

(4) Type of word is also an important predictor, explaining $35 \%$ of the variation in the response.

(5) Type of word elaboration and frequency of occurrence make a smaller contribution to the model: They explain, respectively , $25 \%$ and $24 \%$ of the variation in lexical gain.

As mentioned above, one of the differences found between the two verbs is their subject. While ACCOUNT FOR scarcely takes human entities as subject, EXPLAIN has quite a few instances. An explanation for this difference may result from their different syntactical pattern, as shown in the following examples. It can be seen that the verb EXPLAIN can take that-clause as complement (Examples 6 and 7) and can be used as both transitive (Example 8) and intransitive verbs (Examples 9 and 10). Namely, the flexibility of the use of EXPLAIN may thus account for its more frequent and wide occurrence.

(6) Laufer and Hulstijn (2001) explained that there are similarities between students' behaviors during a reading or writing task (e.g., using dictionaries, writing a composition) and their behaviors during oral interaction tasks.

(7) In the introduction to their book (pp. 4-5), the authors explain that they selected collocations "from those identified as significant by the Cambridge International Corpus of written and spoken English and also the CANCODE corpus of spoken English".

(8) Dijkstra et al. (2005) explain this, in accordance with earlier results from experiments on interlingual homographs (e.g., Dijkstra, Van Jaarsveld, \& Ten Brinke, 1998), by suggesting that this is evidence for cross-linguistic interaction resulting from nonselective lexical....

(9) As Liu (2008) explains, it may not be effective to learn idioms in such lessons because "idioms are best learned naturally in normal language use" (p. 124).

(10) As I will explain, the field that chose the better route is not our own.

In sum, the two verbs under investigation function similarly in the corpus; they are usually used to report the results of regression analysis, where they seem to be interchangeable. The only noticeable difference between them lies in their subject position, which may be explained by the fact that EXPLAIN has various argument structures while ACCOUNT FOR does not. Finally, it should be noted that the current analysis may somewhat be constrained by the low frequency of occurrence of the verb phrase ACCOUNT FOR in the corpus.

\section{ANSWER vS RESPOND}

The lemma ANSWER (i.e., the verb base claim plus its inflected forms tagged as verb) occurs 96 times with a range of 41 in the PA Corpus. The lemma RESPOND, on the other hand, occurs 110 times with a range of 33. All of the concordance lines for ANSWER and 100 randomly-selected concordances lines for RESPOND were examined.

First of all, ANSWER is mostly used as transitive verbs while RESPOND is usually used as intransitive verbs. The subject position for the two verbs is similar, usually human entities such participants or students. Differences, however, were found in their object position. ANSWER almost always collocates with questions or items in the object position while RESPOND (to) collocates with various forms of situations, as shown in the following examples.

(1) For instance, learners would need to correctly answer at least 45 46 of the 48 items that make up the 1000 and 2000 word-frequency levels on the LVLT to comprehend aural texts made up of the first 2000 high.... 
(2) These results partially answer research question 3.

(3) It is difficult to know why these two top-down listeners responded to the course in such contrasting fashion.

(4) I wanted to find out how the children responded to such an approach, whether it resulted in interactions that were less typical of pedagogic discourse, and whether they actually learned any English.

(5) ...; learners may have responded to articles, adjectives, or issues such as semantics that were not intended to be measured at all.

The examples above show that RESPOND attracts a wider range of nouns than ANSWER as the word items in Example 1 is basically another way to say questions. It should be noted that though RESPOND collocates with questions as well, there are no instances of "research questions". It may well be that "answer research questions" is a fixed expression in academic writing.

Secondly, RESPOND attracts a wider variety of adverbs than ANSWER, as shown in the following examples.

(6) Trials for which questions were incorrectly answered and those with reading times 2.5 standard deviations above or below the mean were eliminated for each subject.

(7) ...these analyses provide a complete picture of the whole process and help to fully answer research questions 3 and 2 .

(8) Overall, the means seem to indicate that both groups responded faster as a result of the treatment.

(9) More specifically, do high imagers and low imagers respond differently to this kind of stimulus?

(10) Overall, learners responded positively to the podcast integration....

It was found that ASNWER collocates with adverbs either suggesting (in)correctness or degrees of fulfillment while RESPOND can be modified with various kinds of adverbs. This difference could be attributed to the types of nous they collocate with.

Thirdly, RESPOND seems to be used more frequently in reaction to previous literature than ANSWER. Among the concordance lines examined, there is only one instance for ANSWER but there are three for RESPOND. In the context where the writers used their own research in response to earlier literature, the two verbs seem interchangeable, as shown in the following examples.

(11) Furthermore, we have shown that CA can answer the call by Ellis (2005a) to develop a social account of what participants do when they plan future tasks, explicate how they do this, and discover....

(12) This key finding also responds to earlier critiques (Berne, 2004; Vandergrift, 2004) that carefully controlled studies of this approach are needed to clarify the effects of this approach to teaching....

(13) ..., we believe that the present investigation is the first to appropriately respond to Gardner and Tremblay's (1994) call for empirical tests of the effectiveness of motivational strategies in language classes.

(14) Our study accomplishes this in a longitudinal design, responding to frequent calls in the literature for more research on oral data studied longitudinally (Ortega \& Iberri-Shea, 2005).

In sum, ANSWER and RESPOND exhibit both similarities and differences in terms of their object position and the adverbs that they collocate with. It appears that RESPOND is a generic verb while ANSWER is more specific, and that may be why RESPOND collocates with a wider range of nouns and adverbs than ANSWER in the corpus. Besides, the two verbs can both be used in reaction to calls from previous research, and in this case they seem completely interchangeable though RESPOND is used slightly more frequently. 


\section{Conclusion}

Based on the preliminary analysis, there are some interesting findings for the synonymous verb pairs under investigation in this study. Their syntactical structures and preferred collocates suggest different patterns for these verbs. The results should be able to provide some insights for the expert performance in the academic field of Applied Linguistics. However, this is a preliminary small-scaled study with manual investigation into the concordance lines, future studies should pay more attention to the statistical significance with quantitative analysis to support the qualitative findings. Besides, some in-depth analysis with more focus on the degree and density within a continuum in terms of these attributive verbs are strongly called for in future research.

\section{ACKNOWLEDGEMENT}

The project was supported by the grant from the Ministry of Science and Technology (MOST1042410-H-240-006) in Taiwan. Part of this paper has been demonstrated at JSLS 2016 and TaLC 12 Conferences.

\section{REFERENCES}

Boers, F., \& Lindstromberg, S. (2009). Optimizing a Lexical Approach to Instructed Second Language Acquisition. Basingstoke, UK: Palgrave Macmillan Publications.

Hunston, S. (1995). A corpus study of some English verbs of attribution. Functions of Language, 2, 133-158.

Lewis, M. (2000). Language in the lexical approach. In M. Lewis (Ed.), Teaching collocation: Further development in the lexical approach (pp. 126-154). Croatia: Thomson \& Heinle.

Jabulani, S. (2014). An analysis of the language of attribution in university students' academic essays. South African Journal of Education, 34(3), 1-10.

Webb, S., \& Kagimoto, E. (2011). Learning collocations: do the number of collocates, position of the node word, and synonymy affect learning? Applied Linguistics, 32(3), 259-276.

Woolard, G. (2000). Collocation - encouraging learner independence. In M. Lewis (Ed.), Teaching collocation: Further development in the lexical approach (pp. 28-46). Croatia: Thomson \& Heinle.

Xiao, R. Z. and McEnery, A. M. (2006). Collocation, semantic prosody and near synonymy: A crosslinguistic perspective. Applied Linguistics, 27 (1), 103-129. 